IFIC $/ 12-20$

RM3-TH/12-3

\title{
Predicting leptonic CP violation in the light of Daya Bay result on $\theta_{13}$
}

\author{
D. Melon \\ Dipartimento di Fisica "E. Amaldi" \\ Universitá degli Studi Roma Tre, Via della Vasca Navale 84, 00146 Roma, Italy \\ S. Moris 丹 $^{\circ}$ and E. Peinadd田 \\ AHEP Group, Instituto de Física Corpuscular - C.S.I.C./Universitat de València \\ Edificio de Institutos de Paterna, Apartado 22085, E-46071 València, Spain
}

\begin{abstract}
In the light of the recent Daya Bay result $\theta_{13}^{D B}=8.8^{\circ} \pm 0.8^{\circ}$, we reconsider the model presented in 1 showing that, when all neutrino oscillation parameters are taken at their best fit values of Schwetz et al. 2] and $\theta_{13}=\theta_{13}^{D B}$, the predicted values of the CP phase are $\delta \approx \pm \pi / 4$.
\end{abstract}

The Daya Bay Collaboration has recently contributed to the longstanding question of the magnitude of $\theta_{13}$ releasing their data on the $\bar{\nu}_{e} \rightarrow \bar{\nu}_{e}$ oscillation [3]; they provide a clear evidence of more than $5 \sigma$ deviation from zero of the reactor angle:

$$
\sin ^{2} 2 \theta_{13}=0.092 \pm 0.016 \pm 0.005 .
$$

Evidence of non-zero reactor angle yields to a potentially measurable $\mathrm{CP}$ phase $\delta$ in future neutrino oscillation experiments 4. For this reason, it is important to study the possible predictions for the Dirac phase allowed by flavour models. The purpose of the present letter is to revise the values of the leptonic CP phase $\delta$ implied by the previous result, in the model proposed in [1] and based on the non-abelian discrete symmetry $S_{3}$. The model gives a Fritzsch-like texture for the Majorana neutrinos and, as a consequence of the zeros in the neutrino mass matrix and the Daya Bay result, predicts peculiar values for the CP phase $|\delta| \approx \pi / 4$, contrary to the expectations of many other models which give vanishing or maximal $\delta$ (see [5] for the implications of $\delta= \pm 90^{\circ}$ on the $\mu-\tau$ symmetry after the Daya Bay results).

In the quark sector, Fritzsch-like textures [6] for both the up and down quark mass matrices of the form [7.

$$
M=\left(\begin{array}{ccc}
0 & A & 0 \\
A^{*} & C & B \\
0 & B^{*} & D
\end{array}\right)
$$

give the well known relation $\tan \theta_{12}=\sqrt{m_{1} / m_{2}}$, which predicts the small Cabibbo angle as a consequence of the strong hierarchy in the masses. A texture as in eq.(2) can also be employed for the Majorana neutrino mass matrix; this is a particular case of the class of two-zero texture [8] which, together with the two relations $\Delta m_{a t m}=m_{3}^{2}-m_{1}^{2}$ and $\Delta m_{\text {sol }}=m_{2}^{2}-m_{1}^{2}$, fixes the absolute neutrino mass scale as suggested in [9. Unlike the quark sector, the solar and atmospheric angles can be large due to the fact that in the neutrino sector the hierarchy is not so pronounced.

Although a vast class of Fritzsch-like textures (and their phenomenological consequences) has been already studied in the literature, in the paper of Ref. [1] we have proposed a leptonic model based on the permutation symmetry $S_{3}$ which naturally gives rise to a Fritzsch-type neutrino Majorana mass matrix (and, in addition, to a nearly diagonal charged leptons). In the neutrino sector, the Majorana mass matrix is generated by dimension five [10 and six operators and we obtained a well defined relation between the Dirac phase $\delta$ and the reactor angle $\theta_{13}$ that we want to revised here in the light of eq.(1).

The model was based on $S_{3}$, the group of permutations of three objects, which is the smallest non-Abelian discrete group. $S_{3}$ contains one doublet irreducible representation and two singlets. Here we report only the basic ingredients of our model and we remand the interested readers to the original paper 1 for further details.

The Higgs sector was extended from one $S U(2)_{L}$-doublet to two $S U(2)_{L}$-doublets, $H_{D}=\left(H_{1}, H_{2}\right)$ belonging to a doublet irreducible representation of $S_{3}$ and other two $S U(2)_{L}$ doublets, $H_{S}$ and $H_{S}^{\prime}$, belonging to singlet

\footnotetext{
*Electronic address: meloni@fis.uniroma3.it

${ }^{\dagger}$ Electronic address: morisi@ific.uv.es

‡Electronic address: eduardo@ific.uv.es
} 
representations of $S_{3}$. We also introduced an electroweak scalar singlet $\chi$ which turns out to be relevant to give a non-vanishing electron and muon masses. In order to have nearly diagonal charged lepton mass matrix we assumed two further parity symmetries, so that the global discrete symmetry group of the model is $G=S_{3} \otimes Z_{5} \otimes Z_{2}$. The matter assignment under $G \otimes S M$ is summarized in Tab. I. We have shown that, from the minimization of the potential,

\begin{tabular}{|c||cccc||ccc|c|}
\hline fields & $L_{D}=L_{1,2}$ & $L_{3}$ & $l_{R_{D}}=l_{R_{1,2}}$ & $l_{R_{3}}$ & $H_{D}$ & $H_{S}$ & $H_{S}^{\prime}$ & $\chi$ \\
\hline$S U_{L}(2)$ & 2 & 2 & 1 & 1 & 2 & 2 & 2 & 1 \\
$S_{3}$ & 2 & 1 & 2 & 1 & 2 & 1 & 1 & 1 \\
$Z_{2}$ & + & - & + & - & + & + & - & + \\
$Z_{5}$ & $\omega^{2}$ & $\omega$ & $\omega$ & $\omega^{2}$ & $\omega^{3}$ & $\omega^{4}$ & $\omega^{4}$ & $\omega^{2}$ \\
\hline
\end{tabular}

TABLE I: Matter assignment of the model of Ref.[1].

a possible solution is given by:

$$
\left\langle H_{D}\right\rangle=(v, 0)
$$

After spontaneously symmetry breaking, the charged lepton mass matrix is:

$$
M_{l}=\left(\begin{array}{ccc}
\frac{y_{2}}{\Lambda} v_{S} v_{\chi} & 0 & 0 \\
\frac{y_{1}}{\Lambda} v v_{\chi} & \frac{y_{2}}{\Lambda} v_{S} v_{\chi} & 0 \\
0 & 0 & y_{3} v_{S}
\end{array}\right)
$$

where $v_{S}=\left\langle H_{S}\right\rangle, v_{\chi}=\langle\chi\rangle$. When $v_{\chi}$ is equal to zero only the $\tau$ lepton is massive. The electron and muon masses are generated by the vev of the scalar $\chi$ and are then suppressed by the large scale $\Lambda$. The mass matrix for the charged leptons can be written in terms of the physical lepton masses as:

$$
M_{l}=\left(\begin{array}{ccc}
\sqrt{m_{e} m_{\mu}} & 0 & 0 \\
-m_{\mu}\left(1-\frac{m_{e}}{m_{\mu}}\right) & \sqrt{m_{e} m_{\mu}} & 0 \\
0 & 0 & m_{\tau}
\end{array}\right)
$$

and the squared matrix $M_{l} M_{l}^{\dagger}$ is then diagonalized by:

$$
U_{L}=\left(\begin{array}{ccc}
\frac{1}{\sqrt{1+\frac{m_{e}}{m_{\mu}}}} & -\sqrt{\frac{m_{e}}{m_{\mu}}} \frac{1}{\sqrt{1+\frac{m_{e}}{m_{\mu}}}} & 0 \\
\sqrt{\frac{m_{e}}{m_{\mu}} \frac{1}{\sqrt{1+\frac{m_{e}}{m_{\mu}}}}} & \frac{1}{\sqrt{1+\frac{m_{e}}{m_{\mu}}}} & 0 \\
0 & 0 & 1
\end{array}\right) \approx\left(\begin{array}{ccc}
1 & -0.07 & 0 \\
0.07 & 1 & 0 \\
0 & 0 & 1
\end{array}\right) .
$$

The neutrino masses are generated by non-renormalizable operators of dimension 5 and 6 invariant under the group $G \times S M$. The neutrino mass matrix given in Ref.[1] is as follows:

$$
M_{\nu}=\left(\begin{array}{ccc}
0 & 2 y_{6}^{\nu}\left(v_{S}^{2}+v_{S}^{\prime 2}\right) v_{\chi} / \Lambda & 0 \\
2 y_{6}^{\nu}\left(v_{S}^{2}+v_{S}^{\prime 2}\right) v_{\chi} / \Lambda & \left(y_{2}^{\nu}+y_{3}^{\nu}+y_{4}^{\nu}\right) v^{2} & y_{9}^{\nu} v v_{S}^{\prime} \\
0 & y_{9}^{\nu} v v_{S}^{\prime} & y_{8}^{\nu}\left(v_{S}^{2}+v_{S}^{\prime 2}\right)
\end{array}\right) \equiv\left(\begin{array}{ccc}
0 & b & 0 \\
b & a & c \\
0 & c & d
\end{array}\right),
$$

where $v_{S}^{\prime}=\left\langle H_{S}^{\prime}\right\rangle$. The mass matrix in eq. (7) depends on five real parameters, one of which is related to the Dirac phase. The other four parameters can be fixed using the experimental information from both solar and atmospheric sectors, namely the solar and atmospheric mixing angles and squared mass differences. The model allows for correlations among the angle $\theta_{13}$ and the CP phase $\delta$ that can be easily obtained using the zeros of the Fritzsch texture $[8$.

In Fig. 1 we show the dependence of $\sin ^{2} \theta_{13}$ as a function of $\delta$ as predicted by our model. The solid line represents the $1 \sigma$ correlation when also the other parameters $\left(\theta_{12}, \theta_{23}\right.$ and the solar-to-atmospheric mass differences ratio $\left.\alpha\right)$ are left free to vary in their $1 \sigma$ allowed ranges, whereas the $2 \sigma$ correlation is represented by the dot-dashed line. The dashed line shows the relation between $\theta_{13}$ and $\delta$ when $\theta_{12}, \theta_{23}$ and $\alpha$ are fixed to their best fit values. The result in eq. (1) is enclosed in the horizontal band. For the sake of completeness, we consider two different fits, the one quoted in [2] (left panel), giving:

$$
\begin{array}{cl}
7.41 \times 10^{-5} \mathrm{eV}^{2}<\Delta m_{\text {sol }}^{2}<7.79 \times 10^{-5} \mathrm{eV}^{2} & 2.34 \times 10^{-3} \mathrm{eV}^{2}<\Delta m_{\text {atm }}^{2}<2.59 \times 10^{-3} \mathrm{eV}^{2} \\
0.298<\sin ^{2} \theta_{12}<0.329 & 0.45<\sin ^{2} \theta_{23}<0.58
\end{array}
$$


and the one in [11]:

$$
\begin{array}{cl}
7.32 \times 10^{-5} \mathrm{eV}^{2}<\Delta m_{\text {sol }}^{2}<7.80 \times 10^{-5} \mathrm{eV}^{2} & 2.26 \times 10^{-3} \mathrm{eV}^{2}<\Delta m_{\text {atm }}^{2}<2.47 \times 10^{-3} \mathrm{eV}^{2} \\
0.291<\sin ^{2} \theta_{12}<0.324 & 0.39<\sin ^{2} \theta_{23}<0.5 .
\end{array}
$$

Let us comment first the results in the left panel. We observe that, even considering the $2 \sigma$ uncertainty, the predicted values for $\sin ^{2} \theta_{13}$ are different from zero so that, to a very good accuracy, our model is compatible with deviation from $\theta_{13}=0$ for any value of the $\mathrm{CP}$ violating phase. More remarkably, taking $\sin ^{2} \theta_{13} \sim 0.024$ as indicated by eq. 1 , and all other oscillation parameters to their best fit values quoted in [2], we predict the $\mathrm{CP}$ phase to be $|\delta| \approx \pi / 4$. If, instead, we consider the $1 \sigma$ ranges on the solar and atmospheric parameters (solid line) we get a CP phase only marginally compatible with $\pi / 2$, that is:

$$
-0.53 \pi<\delta<0.53 \pi
$$

The situation is quite different in the right panel, obtained using the results in [11. In fact, the dashed line does not intersect the Daya Bay result, as a consequence of a much smaller atmospheric angle compared to the one quoted in [2]. This implies a more restricted region for $\delta$, not compatible with maximal CP violation:

$$
-0.40 \pi<\delta<0.40 \pi
$$
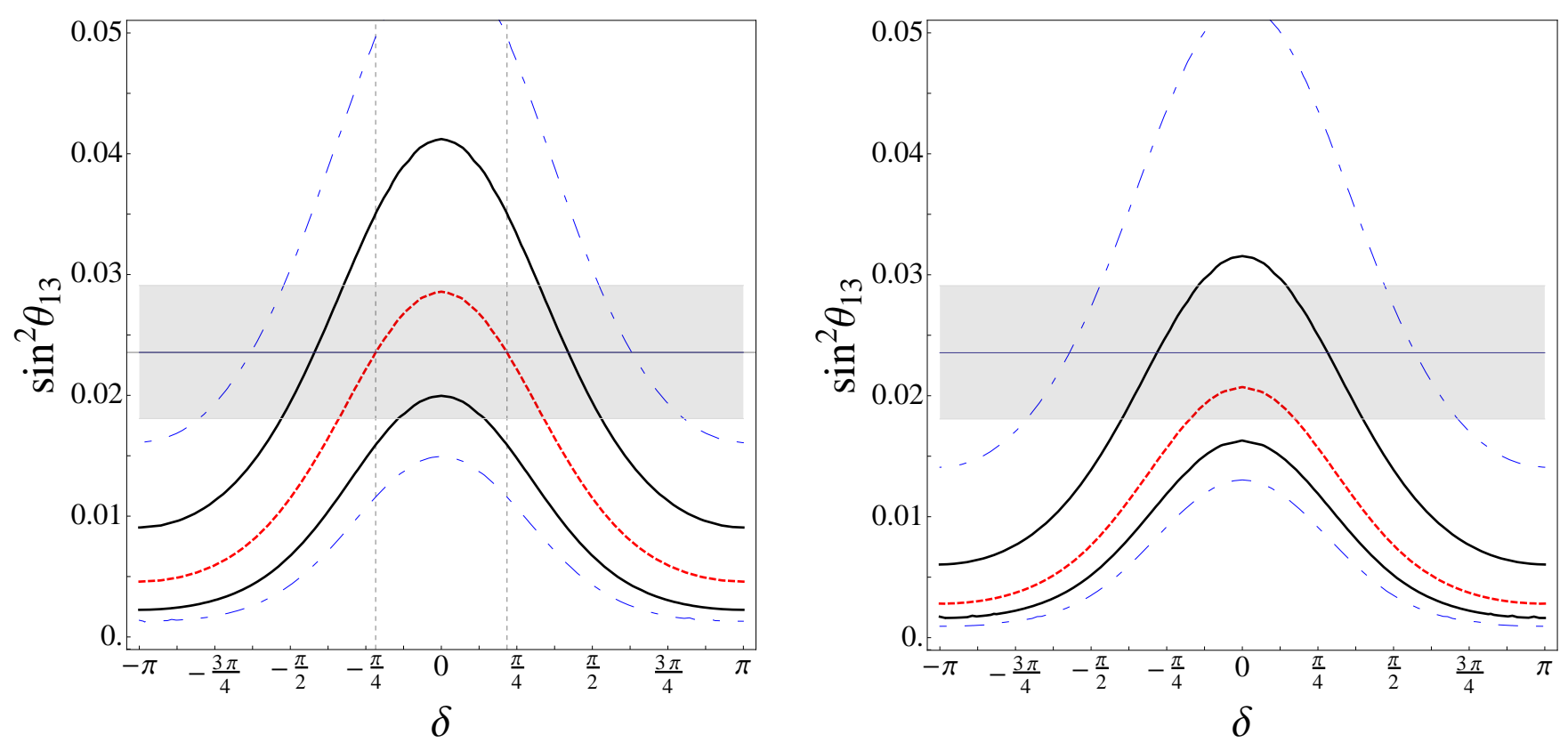

FIG. 1: Correlation among $\delta$ and $\sin ^{2} \theta_{13}$ as obtained in our model, for two different fits, Ref. [2] (left panel) and Ref. [11] (right panel). The $1 \sigma$ result, obtained varying the other oscillation parameters also in their $1 \sigma$ allowed ranges, is shown with solid lines, whereas the $2 \sigma$ result is shown with the dot-dashed line. The dashed line is the relation obtained when $\theta_{12}, \theta_{23}$ and $\alpha$ are fixed to their best fit values. The horizontal band represents the $1 \sigma$ result from Daya Bay [3].

In this paper we have revised the prediction of the model presented in [1] for the leptonic CP phase $\delta$ after the recent result on $\theta_{13}$ given by the Daya Bay experiment. A strong correlation among these two variables is a consequence of the two-zero Fritzsch-texture for the neutrino mass matrix; we have shown that, considering updated values for the solar and atmospheric oscillation parameters, our model predicts a CP phase generally not compatible (or only marginally compatible) with maximal $\mathrm{CP}$ violation. In particular, we get $|\delta| \approx \pi / 4$ if $\theta_{13}$ is taken at the value indicated by Daya Bay and all other mixing parameters as in eq. (8). 


\section{ACKNOWLEDGMENTS}

We thanks M.Hirsch for the useful discussions. Work of SM and EP supported by the Spanish MEC under grants FPA2011-22975 and MULTIDARK CSD2009-00064 (Consolider-Ingenio 2010 Programme), by Prometeo/2009/091

(Generalitat Valenciana), by the EU ITN UNILHC PITN-GA-2009-237920. S. M. is supported by a Juan de la Cierva contract. E. P. is supported by CONACyT (Mexico). D.M. acknowledges MIUR (Italy) for financial support under the program "Futuro in Ricerca 2010 (RBFR10O36O)".

[1] D. Meloni, S. Morisi and E. Peinado, J. Phys. G G 38 (2011) 015003 arXiv:1005.3482 [hep-ph]]

[2] T. Schwetz, M. A. Tortola and J. W. F. Valle, New J. Phys. 10 (2008) 113011 arXiv:0808.2016 [hep-ph]].

[3] F. P. An et al. [Daya Bay Collaboration], "Observation of electron-antineutrino disappearance at Daya Bay"

[4] S. Choubey, R. Gandhi, S. Goswami, J. S. Berg, J. C. Gallardo, H. Kirk, N. Simos and T. Tsang et al., IDS-NF-017.

[5] Z. -z. Xing, arXiv:1203.1672 [hep-ph].

[6] H. Fritzsch, Nucl. Phys. B 155, 189 (1979).

[7] D. s. Du and Z. z. Xing, Phys. Rev. D 48, 2349 (1993): H. Fritzsch and Z. z. Xing, Phys. Lett. B 353, 114 (1995) arXiv:hep-ph/9502297.

[8] P. H. Frampton, S. L. Glashow and D. Marfatia, Phys. Lett. B 536 (2002) 79 arXiv:hep-ph/0201008; Z. z. Xing, Phys. Lett. B 530, 159 (2002) arXiv:hep-ph/0201151; M. Honda, S. Kaneko and M. Tanimoto, JHEP 0309, 028 (2003) arXiv:hepph/0303227; B. R. Desai, D. P. Roy and A. R. Vaucher, Mod. Phys. Lett. A 18, 1355 (2003) arXiv:hep-ph/0209035; W. Grimus and L. Lavoura, J. Phys. G 31, 693 (2005) arXiv:hep-ph/0412283; L. Lavoura, Phys. Lett. B 609, 317 (2005) arXiv:hep-ph/0411232; S. Zhou and Z. z. Xing, Eur. Phys. J. C 38, 495 (2005) arXiv:hep-ph/0404188; W. Grimus, PoS HEP2005, 186 (2006) arXiv:hep-ph/0511078]; H. Fritzsch, Int. J. Mod. Phys. A 24, 3354 (2009) [Int. J. Mod. Phys. A 25, 597 (2010)] arXiv:0906.1066 [hep-ph]]. P. O. Ludl, S. Morisi and E. Peinado, Nucl. Phys. B 857, 411 (2012) arXiv:1109.3393 [hep-ph]].

[9] H. Fritzsch, Int. J. Mod. Phys. A 24 (2009) 3354 [Int. J. Mod. Phys. A 25 (2010) 597] arXiv:0906.1066 [hep-ph]].

[10] S. Weinberg, Phys. Rev. Lett. 43, 1566 (1979).

[11] G. L. Fogli, E. Lisi, A. Marrone, A. Palazzo and A. M. Rotunno, Phys. Rev. D 84, 053007 (2011) arXiv:1106.6028[hep-ph]]. 